\title{
What drives the radio slopes in radio quiet quasars?
}

\author{
Ari Laor ${ }^{1 \star}$, Ranieri D. Baldi ${ }^{2 \star}$ and Ehud Behar ${ }^{1 \star}$ \\ ${ }^{1}$ Physics Department, Technion, Haifa 32000, Israel \\ ${ }^{2}$ School of Physics and Astronomy, University of Southampton, Southampton, SO17 1BJ, UK
}

12 November 2018

\begin{abstract}
The origin of the radio emission in Radio Quiet (RQ) quasars is not established yet. Important hints can be provided by the spectral slope, and its relation to other emission properties. We compiled the highest resolution 5 and $8.4 \mathrm{GHz} V L A$ observations available of the RQ optically selected PG quasars at $z<0.5$. We derive the 5-8.4 GHz spectral slope, $\alpha_{R}$, for 25 of the complete and well studied sample of $71 \mathrm{RQ}$ PG quasars. We find a highly significant correlation of $\alpha_{R}$ with $L / L_{\text {Edd }}$, where high $L / L_{\text {Edd }}(>0.3)$ quasars have a steep slope $\left(\alpha_{R}<-0.5\right)$, indicative of an optically thin synchrotron source. In contrast, lower $L / L_{\mathrm{Edd}}(<0.3)$ quasars generally have a flat slope $\left(\alpha_{R}>-0.5\right)$, indicative of a compact optically thick synchrotron source. Flat $\alpha_{R}$ quasars also have a low $\mathrm{Fe} \mathrm{II} / \mathrm{H} \beta$ line ratio, and a flat soft X-ray slope. The 16 Radio Loud (RL) PGs do not follow the RQ quasar set of correlations, and their $\alpha_{R}$ is set by $M_{\mathrm{BH}}$, suggesting that the radio emission mechanisms in RL and RQ quasars are different. A possible interpretation is that high $L / L_{\mathrm{Edd}} \mathrm{RQ}$ quasars produce a strong outflow and an associated optically thin synchrotron emission. In lower $L / L_{\mathrm{Edd}} \mathrm{RQ}$ quasars, the strong outflow is missing, and only a compact optically thick radio source remains, possibly associated with the accretion disk coronal emission. A systematic study of RQ quasars at higher frequencies, and higher resolution, can test whether a compact flat source indeed resides in the cores of all RQ quasars, and allow the exploration of its relation with the coronal X-ray emission.
\end{abstract}

Key words: quasars: general.

\section{INTRODUCTION}

The radio emission in Radio Quiet Quasars (RQQ) may be produced by a variety of mechanisms. It could be optically thin synchrotron and free-free emission associated with star formation on the host galaxy scale (e.g. Padovani et al. 2011; Condon et al. 2013; Kellermann et al. 2016; Brown et al. 2017; Tabatabaei et al. 2017, cf. White et al. 2015; Zakamska et al. 2016), synchrotron and free-free emission from an AGN driven outflow (e.g. Mundell et al. 2000; Blustin \& Fabian 2009; Jiang et al. 2010; Steenbrugge et al. 2011; Faucher-Giguère \& Quataert 2012; Zakamska \& Greene 2014; Nims, Quataert, \& Faucher-Giguère 2015), optically thick synchrotron emission from a compact source, such as an accretion disk corona (Laor \& Behar, 2008; Raginski \& Laor 2016, hereafter LB08, RL16), or synchrotron emission on various scales from a low power jet (Falcke \& Biermann 1995; Wilson \& Colbert 1995).

The radio spectral slope carries diagnostic power on the emission mechanism. Optically thin free-free has a spectral ^ E-mail: laor@physics.technion.ac.il (AL); R.Baldi@soton.ac.uk
(RDB); behar@physics.technion.ac.il (EB) slope of $\alpha \simeq-0.1\left(=d \log f_{\nu} / d \log \nu\right)$. Optically thin synchrotron emission is characterized by $\alpha<-0.5$. Optically thick free-free emission becomes a blackbody, where $\alpha=2$. Optically thick synchrotron has $\alpha>-0.5$, and can increase up to $\alpha=2.5$ (e.g. Rybicki \& Lightman 1979, RL16). Freefree absorbed synchrotron emission can produce $\alpha>2.5$ (Levinson, Laor, \& Vermeulen 1995; Barvainis \& Lonsdale 1998). Below we define a spectrum as flat when $\alpha>-0.5$, and steep when $\alpha<-0.5$.

A handful of $V L A$ studies of the radio spectral slope of RQQ are available (Antonucci \& Barvainis 1988; Barvainis, Lonsdale, \& Antonucci 1996; Kukula et al. 1998; Barvainis et al. 2005). These studies indicate that close to a half of RQQ show a flat spectrum at 5 to $8.4 \mathrm{GHz}$, suggesting a compact optically thick synchrotron source (a fraction of $40 \%$ is found by Barvainis, Lonsdale, \& Antonucci 1996, using mostly C and D arrays; 7/16 in Kukula et al. 1998, using the A-array; and 5/11 in Barvainis et al. 2005, using various arrays). Optical depth estimates (LB08), and Radiative transfer calculations (RL16), imply that an optically thick source at the given frequencies and luminosities, is smaller than $\sim 0.1-1 \mathrm{pc}$. A handful of nearby X-ray bright RQ AGN with a steep $\alpha$, generally show a spectral flattening towards 
the $\mathrm{mm}$ regime ( $95 \mathrm{GHz}$, Behar et al. 2015, 2018). The flattening at high frequencies indicates that a compact optically thick radio source resides in objects which are dominated at lower frequencies by extended optically thin emission.

A compact radio source on pc scale, or smaller, is also indicated by the handful of $V L B I$ observations of RQQ (Blundell \& Beasley 1998; Caccianiga et al. 2001; Ulvestad, Antonucci, \& Barvainis 2005; Doi et al. 2013; Panessa \& Giroletti 2013), which yield lower limits on the brightness temperature of $T_{B}>10^{8}-10^{9} \mathrm{~K}$, which excludes thermal radio emission. A compact source is also indicated by the observed variability sometimes observed in a handful of sources (Wrobel 2000; Anderson \& Ulvestad 2005; Barvainis et al. 2005; Mundell et al. 2009; Jones et al. 2011; Baldi et al. 2015, cf. Jones, McHardy, \& Maccarone 2017).

A compact flat spectrum optically thick synchrotron source, observed in about half of the RQQ, may in fact be present in all RQQ, if it is an inevitable part of an accretion disk magnetised coronal emission (Field \& Rogers 1993; Di Matteo, Celotti, \& Fabian 1997; Merloni, Di Matteo, \& Fabian 2000). The coronal emission may extend to the radio regime if the magnetised plasma extends to a fraction of a pc, e.g. due to coronal mass ejections (LB08), or due to spatially extended disc corona. Such a compact component produces a flat spectral slope (e.g. RL16), but it apparently dominates the observed radio emission only in about half of RQQ. Why is the radio emission in the other half of RQQ dominated by a more extended optically thin synchrotron source?

Type 1 AGN show a large variety of correlated emission line properties, most notably the so-called eigenvector 1 (EV1) set of correlations (Boroson \& Green 1992 and citations thereafter). High EV1 objects generally have relatively narrow broad lines, specifically narrow $\mathrm{H} \beta$, strong Fe II emission, weak [O III $]$ emission, and asymmetric line profiles with excess blue wing emission. These correlated properties extend to the UV emission lines, with enhanced N V, Si IV+O IV], Si III], C II, and O I line emission, and suppressed C IV emission (Wills et al. 1999). The set of correlations extend to the X-ray regime, where a narrower $\mathrm{H} \beta$ is associated with a steeper soft X-ray slope (Laor et al. 1994; Boller, Brandt, \& Fink 1996; Laor et al. 1997; Grupe et al. 1999; Grupe 2004), and also a steeper hard X-ray slope (Brandt, Mathur, \& Elvis 1997; Reeves \& Turner 2000; Porquet et al. 2004; Piconcelli et al. 2005; Shemmer et al. 2006). A higher EV1 is likely associated with a higher luminosity in Eddington units, $L / L_{\text {Edd }}$ (Boroson \& Green 1992; Sulentic, Marziani, \& Dultzin-Hacyan 2000; Boroson 2002). The physical mechanism which may drive some of the above correlations, is enhanced gas metallicity (Shemmer \& Netzer 2002; Shemmer et al. 2004), and enhanced outflows (Shin, Nagao, \& Woo 2017) in higher $L / L_{\text {Edd }}$ objects.

Is the radio spectral slope in RQQ also part of the EV1 set of correlated properties? If yes, it may provide important hints on their radio emission mechanism. Also, do the radio slopes of RLQ and RQQ show a similar set of correlations? as may be expected if RQQ are scaled down versions of RLQ. To answer these questions, we explore the correlations of the radio spectral slopes and other emission properties of a well studied sample of quasars, and compare the results for identically selected RQQ and RLQ.

Below we find that the radio slope in RQQ is indeed part of the EV1 set of correlations, where narrow $\mathrm{H} \beta$, or equivalently high $L / L_{\text {Edd }}$ objects, have a steeper $\alpha$. In contrast, RLQ do not follow these correlations. Instead, their $\alpha$ gets steeper with increasing black hole mass, $M_{\mathrm{BH}}$. This contrast suggests that RQQ are not just scaled down RLQ. Their radio emission is driven by a different physical mechanism, possibly an outflow which is more prevalent in high $L / L_{\text {Edd }}$ objects.

The paper is organised as follows. In section $\S 2$ we describe the sample and the observations used, in section $\S 3$ we present the results of the correlation analysis, in $\S 4$ we discuss the results and in $\S 5$ provide the main conclusions.

\section{THE SAMPLE}

Our purpose is to derive the spectral slope of the quasar radio emission, excluding as much as possible contamination from the host galaxy radio emission, which may be significant in RQQ. We therefore exclude single dish observations, and use only the highest resolution available $V L A$ observations, preferably with the A configuration. To maximise the angular resolution, we use the higher frequencies which are commonly available, specifically the $\mathrm{C}$ and $\mathrm{X}$ bands at 5 and 8.4 GHz. These criteria provide angular resolutions of 0.4 and 0.24 arcsec respectively. We further select low redshift objects, with a typical $z \sim 0.1$ (see below), which corresponds to physical integration radii of $370 \mathrm{pc}$ and $220 \mathrm{pc}$ respectively. These radii are generally well below the half-light radii of galaxies (e.g. Faber et al. 1997), and we therefore expect that most of the host galaxy radio emission related to stellar processes, is excluded. The observed radial extent of the radio emission in RQQ is indeed generally well below the kpc scale (e.g. Kellermann et al. 1994), suggesting that host contamination is not a major issue. However, star formation contribution to the radio emission on the unresolved sub-kpc core scale may still be present. In some RQQ significant radio emission is present on larger scales, which may be host related, but may also be AGN related. In this study we focus on the unresolved core emission, i.e. below a few hundred pc, and ignore the larger scale resolved emission.

We use the PG quasar sample (Schmidt \& Green 1983), comprised of the brightest 114 quasar in one quarter of the sky, residing above the Galactic plane, and generally above the celestial equator. This sample, although defined 35 years ago, is still the only well define and complete sample of bright quasars, which satisfies two properties which are critical for this study. First, the selection criteria (bright, point-like, blue objects) are independent of the radio properties, thus the radio properties are not directly biased by selection effects. Second, most of the sample $(\sim 90 \%)$ is detected in the radio at $5 \mathrm{GHz}$ (Kellermann et al. 1989). The sample is therefore well defined, unbiased, and nearly complete (Jester et al. 2005). We use the $z<0.5$ subsample of 87 quasars (Boroson \& Green 1992), which was extensively studied over a wide range of wavelengths, and is the prime sample of bright AGN. The extensive data base allows to explore relations of the radio emission with a wide range of emission properties.

Of the 87 objects in the Boroson \& Green (1992) sample, 71 are defined as radio quiet, based on the Kellermann et al. (1989) definition of having $R<10$, where 
$R \equiv f(4400 \AA) / f(6 \mathrm{~cm})$. Of these 71 objects, 62 were detected by Kellermann et al. (1989) at $5 \mathrm{GHz}$ with the D configuration, where the resolution is 14 arcsec, which generally encompasses the entire host emission. Of these $62 \mathrm{ob}-$ jects, 53 were in addition detected at $5 \mathrm{GHz}$ with the high resolution A configuration of 0.4 arcsec. We restrict the sample to the 32 objects with detections at a level of $>5 \sigma$, i.e. a flux $>0.3 \mathrm{mJy}$, as some of the suggested $3 \sigma$ detections in Kellermann et al. (1989) were found to be non detections in the reanalysis of of the same data set by Miller, Rawlings, \& Saunders (1993). The $>5 \sigma$ detection criterion also reduce the error in the derived $5-8.4 \mathrm{GHz}$ spectral slope.

The next step is to search for published $8.4 \mathrm{GHz}$ observations of these 32 objects. Only 15 of the 32 have published detections with the A configuration (Kukula et al. 1995, 1998; Runnoe, Gültekin, \& Rupke 2018). Three additional objects from the sample are part of the $8.4 \mathrm{GHz}$ variability study of Barvainis et al. (2005), using mixed configurations. One additional object was observed in the C/D configurations by Barvainis \& Lonsdale (1997), and five more objects by Barvainis, Lonsdale, \& Antonucci (1996) in the C configuration. Observations of two additional objects are found in the $V L A$ archives, with the A and B configurations, leading to a total of 26 objects with $8.4 \mathrm{GHz}$ observations at al configurations. Of these 26,15 were observed in the A configuration, four in a mixed configuration including $\mathrm{A}$ or $\mathrm{B}$, and seven in the $\mathrm{C}$ and $\mathrm{D}$ configurations. The $\mathrm{B}, \mathrm{C}$ and $\mathrm{D}$ configurations at $8.4 \mathrm{GHz}$ correspond to angular resolutions of 0.7 , 2.5, and 8.6 arcsec, compared to 0.4 arcsec in the A configuration. The derived slope in these objects is therefore subject to a possible bias due to a mismatched aperture effect, as the $5 \mathrm{GHz}$ fluxes of all objects are from the A configuration. To minimise the aperture effect, we checked the value of the core dominance parameter $f_{c}$, defined as the $5 \mathrm{GHz}$ peak flux density in the A configuration to the total $5 \mathrm{GHz}$ flux density in the $\mathrm{D}$ configuration, in the seven objects. We excluded PG 1416-129, where $f_{c}=0.22$ (Kellermann et al. 1989), indicating a dominant extended flux, which implies that the available flux in the $8.4 \mathrm{GHz} \mathrm{D}$ configuration likely includes significant extended flux, which will bias the derived slope. In the other 6 objects $1.43 \geqslant f_{c} \geqslant 0.77$, which indicates a largely compact $5 \mathrm{GHz}$ source, and therefore very likely a compact $8.4 \mathrm{GHz}$ source as well. The available C and D configurations flux in these objects likely does not include significant extended flux, and should provide a good estimate of the core flux. The exclusion of PG 1416-129 leaves a total of 25 objects.

Another possible source of uncertainty in the spectral slope determination, is variability, indicated in three of our objects where $f_{c}>1$. Variability necessarily implies a compact source, less than a fraction of a light year across, given the time difference between the A and D configuration observations (Kellermann et al. 1989). Such a compact source, at the typical $5 \mathrm{GHz}$ luminosity of RQQ, is expected to be optically thick (LB08) and produce a flat spectrum. Indeed, all three objects show a flat slope. Fortunately, the non simultaneity of the 5 and $8.4 \mathrm{GHz}$ observations in these objects did not lead to an apparent steep spectral slope, which is not possible given their compact sizes. A compact variable source may just as well induce a wrong $f_{c}<1$. We cannot exclude a few objects where a steep slope is a variability artefact. To minimise the effect of variability, we use the 5 and $8.4 \mathrm{GHz}$ A configuration observations as close in time as possible. In a few objects where repeated observations indicated significantly (say a factor of $>2$ ) variability, we preferred the closer in time observations in both bands, if available, even from lower resolution configurations.

An alternative definition of radio loudness was suggested by Terashima \& Wilson (2003) based on the radio to X-ray flux ratio $R_{X} \equiv f(1 \mathrm{keV}) / f(6 \mathrm{~cm})$, where RLQ are defined by having $\log R_{X}>-3.5^{1}$. This criterion is consistent with our earlier finding (LB08) that the RQ PG quasars have $\log R_{X} \simeq-5$ with a rather small spread. Three of our RQ PGs, PG 1351+640, PG 1700+518, and PG 2112+059, are RL based on the $\log R_{X}>-3.5$ criterion. However, all three also show significant $\mathrm{C}$ IV absorption (PG $1700+518$ is a BALQ - broad absorption line quasar), and have a steeper $\alpha_{o x}$ than found in unabsorbed quasars (see Fig.4 in Laor $\&$ Brandt 2002). It is therefore possible that both the Xrays and the UV are affected by absorption, which will bias their $R_{X}$ values to be higher than intrinsic. We therefore do not remove these three objects from the RQ sample, despite their having $R_{X}>-3.5$ values.

Table 1 lists the total sample of the 25 RQ PGs where the 5 and $8.4 \mathrm{GHz}$ spectral slope, $\alpha_{R}$, is derived. The table also lists various AGN parameters, including the bolometric luminosity, radio luminosity, $R_{X}$, the $\mathrm{H} \beta$ FWHM, $M_{\mathrm{BH}}$, $L / L_{\mathrm{Edd}}$, the measured 5 and $8.4 \mathrm{GHz}$ fluxes, and $\alpha_{R}$. Also listed is the published ROSAT soft X-ray $(0.2-2 \mathrm{keV})$ slope, $\alpha_{X}$, available for $21 / 25$ of the objects. The various relevant references are detailed in the table notes. Some of the parameters in the table are used below to study their possible correlations with $\alpha_{R}$.

As noted above, in this study we explore only the core emission properties. Eight of the 25 of the objects in the sample have $f_{c}<0.5$ (Kellermann et al. 1989), indicating significant flux on scales larger than 0.4 arcsec, which we do not measure. However, only in two of the eight objects $f_{c}<0.3$. Overall, the core radio emission dominates $\left(f_{c}>\right.$ $0.5)$ in $17 / 25$ of our objects, is comparable $\left(f_{c} \sim 0.5\right)$ to the extended emission in $6 / 25$, and is significantly smaller $\left(f_{c}<0.5\right)$ only in $2 / 25$.

As a comparison sample, we use the 16 RL PGs. These are selected based on exactly the same selection criteria as the 25 RQ PGs. Their emission properties are derived from studies of the PG quasars, irrespective of radio loudness. This again guards against any systematic biases in terms of the derived properties of the RQ versus RL PGs.

Table 2 lists the 16 RL PGs, defined by $R>10$ (Kellermann et al. 1989), and their various emission properties. The interpolated $\alpha_{R}$ near $5 \mathrm{GHz}$ are provided by Falcke, Sherwood, \& Patnaik (1996), based on a compilation of literature values, together with single dish Effelsberg observations. These observations generally include the radio emission integrated over the host galaxy scale or larger, in contrast with the sub kpc integration used above for the RQ PGs. The different integration scales are required, as the RLQ emission generally extends out to large scales. In contrast, in the RQQ sample only the sub kpc scale emission is included,

1 This value is derived by Terashima \& Wilson (2003) for the PG quasars, compared to $\log R_{X}>-4.5$ derived for low luminosity AGN. 
to minimise host contamination. An alternative approach of measuring $\alpha_{R}$ in the RLQ on the same sub kpc scale used for the RQQ, generally reveals only a flat core component.

\section{THE RESULTS}

Figure 1 presents the correlations of $\alpha_{R}$ with the $\mathrm{H} \beta$ FWHM, $L / L_{\mathrm{Edd}}$ and $M_{\mathrm{BH}}$, for the 25 RQ PGs (left hand panels), and for the 16 RL PGs (right hand panels). Note that the three parameters, $\mathrm{H} \beta$ FWHM, $L / L_{\mathrm{Edd}}$ and $M_{\mathrm{BH}}$ are not independent, as $M_{\mathrm{BH}} \propto L^{0.69} \times(\mathrm{H} \beta \mathrm{FWHM})^{2}$ (Davis \& Laor 2011), and $L / L_{\text {Edd }} \propto L^{0.31} \times(\mathrm{H} \beta \text { FWHM })^{-2}$. So, there are only two independent parameters, either $L$ and $\mathrm{H} \beta$ FWHM, or equivalently their linear combination in log space, $M_{\mathrm{BH}}$ and $L / L_{\mathrm{Edd}}$. A highly significant correlation of $\alpha_{R}$ with the $\mathrm{H} \beta \mathrm{FWHM}$, or equivalently with $L / L_{\mathrm{Edd}}$, is present in the RQ PGs, where $\alpha_{R}$ gets steeper with decreasing $\mathrm{H} \beta$ FWHM, or with increasing $L / L_{\mathrm{Edd}}$. The Spearman rank order correlation coefficient is $r_{\mathrm{S}} \simeq 0.67-0.7$ for both correlations, with a probability of being random of $p \simeq(1-3) \times 10^{-4}$ (using the two tailed t-test). The strong correlation of $L / L_{\mathrm{Edd}}$ with $\alpha_{R}$ does not necessarily result from the similarly strong correlation of the $\mathrm{H} \beta$ FWHM with $\alpha_{R}$, together the tight dependence of $L / L_{\mathrm{Edd}}$ on the $\mathrm{H} \beta$ FWHM. This is evident from the significantly weaker correlation of $M_{\mathrm{BH}}$ with $\alpha_{R}$ (lower left panel), where only a marginal trend exist $(p=0.05)$, despite the same strong dependence of $M_{\mathrm{BH}}$ on the $\mathrm{H} \beta$ FWHM.

In contrast with the above correlations for the RQ PGs, the RL PGs show different relations. There is only a weak trend of increasing $\alpha_{R}$ with decreasing $\mathrm{H} \beta$ FWHM, and with increasing $L / L_{\text {Edd }}$ (significance $p=0.08-0.11$ ), which is the opposite of the relations found in the RQ PGs. However, a significant correlation is present between $\alpha_{R}$ and $M_{\mathrm{BH}}$ $\left(r_{\mathrm{S}}=-0.6856, p=0.0034\right.$, i.e $\left.>3 \sigma\right)$, of decreasing $\alpha_{R}$ with increasing $M_{\mathrm{BH}}$, in contrast with the marginal and opposite trend followed by the RQ PGs.

Figure 2 presents correlations of some additional emission properties of the RQ PGs with $\alpha_{R}$. Of all the optical emission line parameters published by Boroson \& Green (1992), the strongest correlation is found between $\alpha_{R}$ and the Fe II $/ \mathrm{H} \beta$ line ratio (upper left panel, $r_{\mathrm{S}}=-0.620, p=$ $0.001)$. A flat $\alpha_{R}(>-0.5)$ is always associated with a low Fe II $/ \mathrm{H} \beta(<0.4)$, while a steep $\alpha_{R}(<-0.5)$ is associated with a wide range of $\mathrm{Fe} \mathrm{II} / \mathrm{H} \beta$ values. Similarly, a high $\mathrm{Fe} \mathrm{II} / \mathrm{H} \beta(>0.6)$ is found only in steep $\alpha_{R}(>-0.5)$ objects. A marginally significant correlation is present between $\alpha_{R}$ and the $\mathrm{H} \beta$ emission line asymmetry (lower left panel, $\left.r_{\mathrm{S}}=-0.476, p=0.0162\right)$, where the steep $\alpha_{R}$ objects tend to have positive asymmetry, i.e. excess blue wing flux (Boroson \& Green 1992).

Although the $\mathrm{Fe}$ II $/ \mathrm{H} \beta$ correlation with $\alpha_{R}$ is significant, it is not independent of the above $\alpha_{R}$ versus $L / L_{\mathrm{Edd}}$ correlation, as Fe II $/ \mathrm{H} \beta$ and $L / L_{\text {Edd }}$ are strongly correlated $\left(r_{\mathrm{S}}=0.777\right)$. Indeed, a partial correlation analysis of $\mathrm{Fe} \mathrm{II} / \mathrm{H} \beta$ with $\alpha_{R}$, at a fixed $L / L_{\mathrm{Edd}}$, is insignificant $\left(r_{\mathrm{S}}=0.176\right)$. Similarly, the weaker correlation of the $\mathrm{H} \beta$ asymmetry with $\alpha_{R}\left(r_{\mathrm{S}}=-0.476\right)$, becomes insignificant at a fixed $L / L_{\mathrm{Edd}}$. This suggests there is a single physical mechanism which underlies the relation of $\alpha_{R}$ with $L / L_{\mathrm{Edd}}$ and the other quantities, Fe II $/ \mathrm{H} \beta$ and the $\mathrm{H} \beta$ asymmetry, which are tightly related with $L / L_{\mathrm{Edd}}$.

Figure 2, upper right panel, explores the relation of $\alpha_{R}$ and $\alpha_{X}$ - the soft X-ray spectral slope, as measured by ROSAT at $0.2-2 \mathrm{keV}$. The value of $\alpha_{X}$ is available for 21 of the objects (see compilation of references in the notes to Table 1). The $\alpha_{R}$ versus $\alpha_{X}$ relation is interesting to explore as $\alpha_{X}$ is also known to be correlated with $L / L_{\mathrm{Edd}}$ (Laor et al. 1997; Grupe 2004; Porquet et al. 2004; Shemmer et al. 2006). A marginally significant correlation is present between $\alpha_{R}$ and $\alpha_{X}\left(r_{\mathrm{S}}=0.418, \mathrm{p}=0.0589\right)$, where the flat $\alpha_{R}$ objects have a flat $\alpha_{X}(>-1.65)$. A steep $\alpha_{R}$ is, however, associated with the full range of observed $\alpha_{X}$ values. A marginally significant correlation is also present between $\alpha_{X}$ and $L / L_{\mathrm{Edd}}\left(r_{\mathrm{S}}=-0.443\right)$, which is not as strong as the correlation of $\alpha_{R}$ with $L / L_{\mathrm{Edd}}\left(r_{\mathrm{S}}=-0.695\right)$ found here.

Figure 2, lower right panel, shows there is no statistically significant relation between $\alpha_{R}$ and the core dominance parameter, $f_{c}\left(r_{\mathrm{S}}=-0.077\right)$. In contrast, the $16 \mathrm{RL}$ PGs show a very strong correlation $\left(r_{\mathrm{S}}=0.726\right)$. Specifically, $4 / 5$ of the flat spectra RL PGs have $f_{c}>0.7$, while all 11 steep spectra objects have $f_{c}<0.4$. This relation implies that an unresolved source, i.e. a source smaller than a fraction of a $\mathrm{kpc}$, is generally an optically thick synchrotron source, and the emission actually comes from a region smaller than a few pc. An extended source is always optically thin (steep slope), as expected. The tight $\alpha_{R}$ versus $f_{c}$ relation generally holds in radio selected RLQ, and is interpreted as a combination of beaming and inclination effects, of otherwise similar objects. However, in contrast with radio selected AGN, the RL PGs are selected independently of their radio properties, and it is not clear that their observed distribution of radio properties can be explained by only beaming and inclination, rather than a distribution of intrinsic properties.

Note that despite the lack of an $\alpha_{R}$ versus $f_{c}$ correlation in the RQ PGs, the four objects with $f_{c}>$ 1.1, PG 1011-040, PG 1216+069, PG 1351+640, and PG 2304+042, i.e. the objects which are inevitably variable, and therefore necessarily compact, do tend to be on the flat $\alpha_{R}$ side.

There is an equal number of RQ PGs with steep and flat core emission of the eight objects at $f_{c}<0.5$. This may well be a true, rather than variability effect on $f_{c}$, as the core can still be extended only to a fraction of $\mathrm{kpc}$, and be dominated by optically thin synchrotron source. The main difference between RLQ and RQQ is that in RLQ the core emission is generally $\mathrm{flat}^{2}$, while in the RQ PGs, the core can be either steep or flat.

\subsection{VLBI observations}

Important input concerning the nature of the radio emission is provided by high resolution $V L B I$ observations of the mas scale emission. Although extensive $V L B I$ studies are available for nearby AGN (e.g. Middelberg et al. 2004 and references therein), most of the objects observed either have very low accretion rates $\left(\log L / L_{\text {Edd }}<-2\right)$, are obscured AGN, or are RL AGN. Only a handful of $V L B I$ studies include

2 excluding the small sub-class of compact steep spectrum objects, see O'Dea (1998). 
RQQ, and only a couple of these studies (Ulvestad, Antonucci, \& Barvainis 2005; Panessa \& Giroletti 2013) provide observations in more than one band, required to measure the spectral slope, $\alpha_{\text {mas }}$, of the mas scale emission. Ulvestad, Antonucci, \& Barvainis (2005) provide multifrequency observations for four RQQ. Two belongs to our sample, PG $1216+069$ which has $\alpha_{\text {mas }}=-0.13$, and PG $1351+640$ with $\alpha_{\text {mas }}=-0.96$, measured between 2.27 and 5 GHz. One of the two other sources is a bright Seyfert galaxy (Mrk 817), with $\alpha_{\text {mas }}=-0.60$ between 1.7 and 5 GHz. This AGN is actually listed in the PG catalog (PG 1434+590, Green, Schmidt, \& Liebert 1986) but is excluded from the final PG quasar sample based on the lack of a quasi-stellar morphology (being host dominated). Based on Table 1 in Vestergaard \& Peterson (2006) it has $\log L / L_{\mathrm{Edd}}=-0.83$, which is typical in our sample. We therefore include this object in this analysis. The fourth object is IRAS $07598+6508$, which also has a high $L / L_{\mathrm{Edd}}$ (see below). It has $\alpha_{\text {mas }}=-1.23$ at $1.67-5 \mathrm{GHz}$. This is a low ionisation BALQ (Boroson \& Meyers 1992), which is likely highly absorbed. The $V L A \mathrm{C} / \mathrm{D}$ configuration gives $\alpha_{R}=-1.41$ (Barvainis \& Lonsdale 1997). We estimate its $L / L_{\text {Edd }}$ based on the $\mathrm{H} \alpha$ FWHM $\left(2550 \mathrm{~km} \mathrm{~s}^{-1}\right)$, and $5000 \AA$ luminosity (Boroson \& Meyers 1992), and the standard expressions (Vestergaard \& Peterson 2006), which together give $\log M_{\mathrm{BH}}=8.18$ and $\log L / L_{\mathrm{Edd}}=-0.28$, again typical in our sample.

Panessa \& Giroletti (2013) provide $\alpha_{\text {mas }}$ for 23 nearby Seyfert galaxies, most of which are of extremely low $L / L_{\text {Edd }}$, or are type 2 AGN. The only type 1 AGN in their study with $\log L / L_{\mathrm{Edd}}>-2$ is NGC 4151 , where $\log L / L_{\mathrm{Edd}}=-1.64$ (Vestergaard \& Peterson 2006), and $\alpha_{\text {mas }}=0.25$ at 1.7 $5 \mathrm{GHz}$.

We also find two non simultaneous $V L B I$ observations at different bands for PG $1700+518$. Blundell \& Beasley (1998) find a flux of $0.8 \mathrm{mJy}$ with the $V L B A$ at $8.4 \mathrm{GHz}$, and Yang et al. (2012) measure a flux of $1.9 \mathrm{mJy}$ at $1.6 \mathrm{GHz}$ using the European VLBI Network, which together imply $\alpha_{\text {mas }}=-0.52$ at $1.6-8.4 \mathrm{GHz}$.

Figure 3 compares the position of the above 6 objects in the $\alpha_{\text {mas }}$ versus $L / L_{\mathrm{Edd}}$ plane, compared to the distribution of $\alpha_{R}$ versus $L / L_{\mathrm{Edd}}$ derived earlier. A dotted line connects $\alpha_{\text {mas }}$ and $\alpha_{R}$ in the three objects which are also part of our $V L A$ sample. Although the $V L B I$ sample is small, the two distributions appear to generally overlap. The one somewhat deviant object is $\mathrm{PG} 1351+640$, with a rather steep slope of $\alpha_{\text {mas }}=-0.96$, despite its low $L / L_{\mathrm{Edd}}$. This is a rather highly variable object, which varies by a factor of 4 within 3 years at 5 and $15 \mathrm{GHz}$ (see Fig. 3 and table 7 in Barvainis \& Antonucci 1989). It is a marginally RLQ, with $R=4.32$ (Kellermann et al. 1989), and in fact reaches $R>10$ at the high state. This large amplitude variability is generally not seen in RQQ (Barvainis et al. 2005). So PG 1351+640 appears to be an intermediate object between RQQ and RLQ.

The fraction of the $5 \mathrm{GHz} V L A$ A configuration core flux $(<0.4$ arcsec), which remains unresolved on the $V L B I$ mas scale, is in the range of $0.5-1$, indicating that most of the core flux is from a compact source on a pc scale, rather than an extended source on the sub-kpc scale.

\section{DISCUSSION}

The main result of our study of the $5-8.4 \mathrm{GHz}$ radio core $(<0.4$ arcsec) emission of 25 RQ PGs, is the correlation of $\alpha_{R}$ and $L / L_{\mathrm{Edd}}$. Specifically, nearly all RQQ in our sample with $L / L_{\text {Edd }}<0.3$ show a flat slope $\left(\alpha_{R}>-0.5\right)$, and all RQQ with $L / L_{\mathrm{Edd}}>0.3$, show a steep slope $\left(\alpha_{R}<-0.5\right)$.

\subsection{Earlier Studies}

Earlier hints for the $\alpha_{R}$ versus $L / L_{\text {Edd }}$ relation can be found in Barvainis, Lonsdale, \& Antonucci (1996), who noted that BALQs, which are believed to have a high $L / L_{\text {Edd }}$ (e.g. Boroson 2002), generally have a steep radio slope. In a followup study of the radio slope of BALQs, Barvainis \& Lonsdale (1997) refute this earlier finding, as they find some flat spectra BALQs. Their discrepant objects are, PG 1416-129, which Green et al. (1997) found was misclassified as BALQ, and UM 275 and Mrk 231, which are both RLQ (have $R>10$, based on fluxes from NED), and are therefore not expected to follow the $\alpha_{R}$ versus $L / L_{\text {Edd }}$ relation. Thus, the results of Barvainis \& Lonsdale (1997) support, rather than refute, the earlier suggestion of Barvainis, Lonsdale, \& Antonucci (1996) that RQ BALQs have a steep radio slope.

Another hint can be found in a conference proceeding of Moran (2000), who noted that narrow line Seyfert 1 galaxies, which are generally high $L / L_{\mathrm{Edd}} \mathrm{AGN}$, tend to have a steep radio slope. In addition, Hwang et al. (2018) recently found that extremely red quasars (ERQs) are characterised by a steep radio slope. The ERQs are extreme luminosity quasars, with a median $L_{\text {bol }} \simeq 10^{47} \mathrm{erg} \mathrm{s}^{-1}$, and are thus very likely high $L / L_{\text {Edd }}$ quasars, as also indicated by their emission line properties (Hamann et al. 2017). Thus, the $\alpha_{R}$ versus $L / L_{\mathrm{Edd}}$ relation may hold over a wide range of luminosities, from the nearby Seyferts level to the most luminous high redshift quasars.

A potentially related result is that very low luminosity AGN with $L / L_{\mathrm{Edd}} \ll 10^{-2}$, generally have a flat radio slope (see Ho 2008 and references therein). This may imply that the relation found here of a flat $\alpha_{R}$ down to $L / L_{\mathrm{Edd}} \simeq 10^{-1.5}$, may extend to much lower $L / L_{\mathrm{Edd}}$.

\subsection{Implications}

The direct physical implication of the $\alpha_{R}$ versus $L / L_{\mathrm{Edd}}$ relation is that the $5-8.4 \mathrm{GHz}$ synchrotron source in lower $L / L_{\text {Edd }}$ AGN is optically thick, and in higher $L / L_{\text {Edd }}$ AGN is optically thin. An optically thick source at typical RQQ radio luminosities implies a physical size $<0.1$ pc (e.g. LB08, eq. 22 there), which indicates the radio originates on the accretion disk scale. The relativistic electrons and magnetic fields which produce the synchrotron radiation, may be produced locally by an outer accretion disc coronal activity, by coronal mass ejections (CME) from the inner accretion disc corona, or may be associated with the base of a nonrelativistic compact jet, launched from the accretion disk.

In contrast, synchrotron emission which is optically thin at $5-8.4 \mathrm{GHz}$ indicates a source larger than $\sim 0.1 \mathrm{pc}$. The source can be as large as a few hundred pc, which would remain unresolved at our angular resolution of a fraction of an arcsec, for our typical $z \sim 0.1$ objects. An extended optically thin source can be produced by expanding blobs of 
magnetised relativistic electrons, produced by CME events from the innermost disc. The ejected blobs will be optically thick on small scales, and will inevitably become optically thin as they move out, expand, and cool. The question is whether they will remain powerful enough to dominate the emission. Such large scale blobs may also be ejected in a jet. The jet needs to be significantly sub-relativistic, given the observed constraints on the proper motion of the emission features on the pc scale (Middelberg et al. 2004; Ulvestad et al. 2005).

\subsection{Outflow?}

An extended synchrotron source may also be produced by an AGN driven wind interaction with the ambient ISM, and the associated shock acceleration. Current such modelling for the radio emission in RQQ (Jiang et al. 2010; FaucherGiguère \& Quataert 2012; Nims, Quataert, \& FaucherGiguère 2015) address a more extended, kpc scale AGN driven wind, which interacts with the host ISM. However, given the significant modelling uncertainties, similar scaled down models of a sub-kpc wind interaction with ambient gas, may also provide a solution consistent with observations. Such a nuclear wind may extend out with detectable emission to larger scales. Indeed, radio images of objects with resolved radio emission often shows a morphology on the host galaxy scale which appears as an extension of the core emission, rather than a morphology which follows the host morphology (Kellermann et al. 1989; Miller, Rawlings, \& Saunders 1993; Gallimore et al. 2006). Is the presence of resolved host scale radio emission related to the presence of unresolved optically thin core emission? Such a relation is expected if both are driven by a given nuclear AGN powered wind which extends from the sub-kpc to the kpc scale.

The optically thin radio emission is observed almost exclusively at high $L / L_{\mathrm{Edd}}$, do these objects preferentially show host scale radio emission? Of the 14 RQ PGs with $L / L_{\text {Edd }}>0.3,13$ show steep $\alpha_{R}<-0.5$ emission, of which $9(=69 \%)$ have $f_{c} \leqslant 0.77$, indicating significant emission outside the core region, i.e. on scales larger than a few hundred pc. However, a similar fraction of $7 / 11(=64 \%)$ of the flat spectrum RQ PGs also have $f_{c} \leqslant 0.77$. Thus, the presence of significant emission on the host galaxy scale is unrelated to $L / L_{\mathrm{Edd}}$ and to the core $\alpha_{R}$. A lack of a relation is also suggest by the absence of a significant correlation between $f_{c}$ and $\alpha_{R}$ (Fig. 2, lower right panel). Steep slope $\left(\alpha_{R}<-0.5\right)$ core emission is found at all values of $f_{c}$. Similarly, the lowest values of $f_{c} \sim 0.3$ is found at all values of $\alpha_{R}$. Thus, if the core steep $\alpha_{R}$ emission is due to a wind/jet, then this outflow is not related to the extended host scale emission.

\subsection{Star Formation?}

Another source for steep core emission is sub-kpc star formation activity. Indeed, a high $L / L_{\text {Edd }}$ may be associated with a higher specific star formation rate (SFR) (Sani et al. 2010; Heckman \& Best 2014). However, can the observed level of the radio luminosity from the core be produced by plausible levels SFR? The observed radio luminosity versus SFR relation (e.g. Brown et al. 2017; Tabatabaei et al. 2017) and the typical $L_{\mathrm{R}} \sim 10^{39}-10^{40} \mathrm{erg} \mathrm{s}^{-1}$ in our sample (Table 1, extended with $\alpha=-1$ to $1.4 \mathrm{GHz}$ ) implies a star formation rate of $10-100 \mathrm{M}_{\odot} \mathrm{yr}^{-1}$ (Brown et al. 2017, Fig.12 there), or 40-400 $\mathrm{M}_{\odot} \mathrm{yr}^{-1}$ (Tabatabaei et al. 2017, eq.16 there). This SFR should be confined typically to $<0.3 \mathrm{kpc}$ from the centre (to remain unresolved with the $V L A$ ), which implies a rather extreme SFR $>100-1000 M_{\odot} \mathrm{kpc}^{-2} \mathrm{yr}^{-1}$ at the core. The Kennicutt-Schmidt law implies an associated surface gas density (e.g. Kennicutt \& Evans 2012, Fig.11 there) of $>10^{4}-10^{5} M_{\odot} \mathrm{pc}^{-2}$, or a total associated gas mass $M_{\text {gas }}>3 \times 10^{10}-3 \times 10^{11} \mathrm{M}_{\odot}$ at the core. Can such a high $M_{\text {gas }}$ reside at the core? The implied associated Keplerian velocity on this scale is $V \sim \sqrt{G M / R}>700-2000 \mathrm{~km} \mathrm{~s}^{-1}$, which can generally be ruled out by the narrow lines emitted on this scale. The expected continuum emission associated with the $\mathrm{SF}$ at other bands, and the various narrow emission lines luminosities associated with the above SFR (Tabatabaei et al. 2017; Brown et al. 2017), may also allow exclusion of such extremely high SFR at the core of RQQ (e.g. Zakamska \& Greene 2014).

\subsection{Are RQQ a scaled down version of RLQ jets?}

Possibly the simplest interpretation of RQQ is that their radio emission is also powered by a relativistic jet, as in RLQ, but their jet power is scaled down by a factor of $\sim$ 1000. In RLQ $\alpha_{R}$ depends on orientation due to relativistic beaming, where edge-on RLQ, with a jet side-view, are steep spectrum lobe dominated, and face-on RLQ, with a head-on view of the jet, are generally flat spectrum core dominated, e.g. Wills \& Browne (1986). Could $\alpha_{R}$ in RQQ also be set by inclination? If true, then face on flat $\alpha_{R}$ RQQ are expected to have a narrower $\mathrm{H} \beta \mathrm{FWHM}$, due to a face-on view of the broad line region as well, and the steep $\alpha_{R}$ objects will tend to show a broader $\mathrm{H} \beta$ FWHM due to the edge on view, as observed in RLQ (Wills \& Browne 1986). However, as shown in Fig.1, the RQ PGs show the opposite correlation, where flat $\alpha_{R}$ RQQ, presumably face-on, show broader rather than narrower lines. This excludes jet collimation together with orientations as the origin of the $\alpha_{R}$ correlations in the RQ PGs.

Although orientation is ruled out, a scaled down nonrelativistic jet which emits isotropically, is not excluded. However, why does $\alpha_{R}$ from a weak non-relativistic jet shows the correlations found here is not addressed by this scenario.

Since RLQ are generally derived from radio selected samples, while RQQ from optically selected samples, the observed differences may just reflect the different sample selection criteria, of otherwise similar objects. To overcome this bias, one needs to compare RLQ and RQQ with the same selection criteria. The RL and RQ PGs selection criteria are the same, and therefore the different $\alpha_{R}$ correlations in the two populations (Fig.1), provides strong evidence for significantly different radio emission mechanisms. The RL PGs show a significant correlation of $\alpha_{R}$ with $M_{\mathrm{BH}}$, while the RQ PGs show a marginal and opposite trend. The high $M_{\mathrm{BH}}$ RL PGs generally show a steep slope spatially extended lobe emission. If a similar scaled down mechanism existed in the RQ PGs, they would have shown the same trend of $\alpha_{R}$ with $M_{\mathrm{BH}}$ as the RL PGs, even if the lobes were compact and spatially unresolved.

Similarly, the correlation of $\alpha_{R}$ with $L / L_{\mathrm{Edd}}$ in the RQ 
PGs is absent in the RL PGs (with a hint of a reverse trend). The RQ PGs at low $L / L_{\text {Edd }}$ do not produce an extended optically thin radio source. In contrast, in RLQ, jet launching and extended radio emission is prevalent at low $L / L_{\text {Edd }}$. Again pointing at a different radio production mechanisms in RL and RQ quasars.

\subsection{How compact is the optically thin source?}

A direct measurement of the size of the unresolved core component is provided by comparing the mas scale flux measured with the $V L B I$, with the arcsec flux measured with the $V L A$. In flat spectrum objects the flux generally originates on the mas scale (see Orienti \& Prieto 2010; Middelberg et al. 2004), as theoretically expected for an optically thick source. But, how compact is the radio source in the steep spectrum objects? Six of our RQ PGs were observed by Blundell \& Beasley (1998) with the VLBA using a single band at $8.4 \mathrm{GHz}$, of which five show a steep spectrum with the $V L A$. In one steep slope object $(\mathrm{PG} 1700+517)>90 \%$ of the $V L A$ flux remains unresolved on the mas scale, while in the other five objects the mas includes $<50 \%$ of the flux.

Two of the steep spectra objects, PG $1216+069$ and PG $1351+640$, were also observed by Ulvestad, Antonucci, \& Barvainis (2005) with the $V L B A$ at $5 \mathrm{GHz}$, who find that the mas includes $70 \%$ and $97 \%$ of the $V L A$ flux, in contrast with fractions of $14 \%$ and $43 \%$ found by Blundell \& Beasley (1998) at $8.4 \mathrm{GHz}$. These rather discrepant results reflect the fact that both objects are variable (both have $f_{c}>1$ ). This demonstrates the potential difficulty in comparing non simultaneous $V L A$ and mas observations. The presence of significant variability already shows that the radio source is compact in both objects, and is consistent with their high $f_{c}$ values.

We conclude that the steep component may be as compact as the pc scale (PG 1216+069, PG 1351+640, PG 1700+517), but potentially a significant fraction originates on larger scales. Clearly, a larger systematic study of the steep spectra quasars on both the mas and arcsec scale is required to get better constraints on the spatial extent of the steep spectrum source.

Does the $L / L_{\text {Edd }}$ versus $\alpha_{R}$ correlation extends to the mas scale? The literature data we compiled (Fig.3) on six type 1 AGN with $\log L / L_{\text {Edd }}>-2$, of which three belong to the PG sample, suggests (with significant scatter) that the slope versus $L / L_{\mathrm{Edd}}$ relation may hold on the pc scale. Multi-band $V L B I$ observations of a large and well defined sample of RQQ is required to properly address this physically interesting question.

The high brightness temperature of the mas emission, typically well above $10^{8} \mathrm{~K}$ (Ulvestad, Antonucci, \& Barvainis 2005), clearly excludes a thermal free-free origin of the compact pc scale radio emission. Similarly, variability, which is observed in some objects, implies a compact sub pc scale source. The presence of variability appears to be similar in amplitude in both steep and flat slope objects (Barvainis et al. 2005), which suggests that the optically thin sources are also on sub pc scale, and only slightly larger than the optically thick sources. However, the variability detection is generally only marginal, and systematic high quality studies of larger samples are required for more robust conclusions.

\subsection{What are the additional correlations telling us?}

Additional hints are provided by the correlations of $\alpha_{R}$ and the two emission line parameters, the Fe II/H $\beta$ line ratio and the $\mathrm{H} \beta$ asymmetry parameter (Fig. 2 left panels). As noted above (section 3 ), these correlations are not independent of the $\alpha_{R}$ versus $L / L_{\text {Edd }}$ correlation, as both parameters are part of the EV1 set of correlations (Boroson \& Green 1992). High EV1 objects are characterised by a narrow $\mathrm{H} \beta$, and thus a high $L / L_{\mathrm{Edd}}$, high values of $\mathrm{Fe} \mathrm{II} / \mathrm{H} \beta$, and blue excess asymmetry in the $\mathrm{H} \beta$ line profile.

What are these correlations telling us? A physical mechanism which may tie together all these trends is a radiation pressure driven wind. The strength of such a wind depends on the ratio of radiation pressure to gravity, i.e. $L / L_{\mathrm{Edd}}$. The radiation is coupled to the gas through the metals opacity, and is thus expected to scale as the Fe II/H $\beta$ flux ratio, which is likely a metallicity indicator (Shemmer \& Netzer 2002). The blue excess emission of $\mathrm{H} \beta$ may indicate an outflowing component, which is optically thick and obscures the receding wind component. Finally, this outflowing component may either shock, or advect outwards magnetised plasma, which produces the extended optically thin steep $\alpha_{R}$ emission.

A relation may also exists between $\alpha_{R}$ and $\alpha_{X}$ (Fig.2, upper right panel). The flat $\alpha_{R}$ objects all have $\alpha_{X}>-1.65$, while the steep $\alpha_{R}$ objects show a spread of $\alpha_{X}$ values. We note that the X-ray spectral quality for some of the objects is rather low, and some of the $\alpha_{X}$ values may be significantly uncertain, or be affected by absorption.

Why does a compact optically thick radio source tend to be associated with a flat $\alpha_{X}$ ? One may raise the following scenario. If the X-ray power-law is produced by an optically thin thermal comptonizing hot corona (as commonly assumed), then a flatter slope is produced by a hotter corona with a larger optical depth (Rybicki \& Lightman 1979 , eq.7.45b there). If the corona is heated by magnetic reconnection events, and is depleted by CME, wind, or jet, then the lack of an outflow may lead to a hotter and larger optical depth corona, which produces a flatter $\alpha_{X}$. Such a corona also produces a flatter $\alpha_{R}$, as the larger column magnetised plasma is more optically thick. A CME/wind/jet powered by the corona, may lead to a cooler and lowercolumn corona, and thus a steeper $\alpha_{X}$, while the ejected magnetised plasma will produce an optically thin steeper $\alpha_{R}$.

\subsection{Future followups}

This study is based on an archival study of non-simultaneous observations of 25 of the 71 RQ PGs. Clearly, a systematic study of $\alpha_{R}$, based on simultaneous observations of a complete and well defined sample, is warranted. The PG sample is a natural place to start. This will allow the exploration of how robust are the empirical relations found here, and whether additional significant relations exist between $\alpha_{R}$ and the observed wide range of quasar emission properties. In particular, is $\alpha_{R}$ part of the EV1 set of properties, as suggested in this study?

Does the $\alpha_{R}$ versus $L / L_{\text {Edd }}$ relation extends to ex- 
tremely low $L / L_{\text {Edd }}$ nearby AGN? does it hold in luminous high $z$ AGN?

Do all RQQ harbour a compact flat spectrum component, as expected if magnetised plasma is always associated with coronal activity? If yes, then the flat component may become dominant at a high enough frequency also in the steep $\alpha_{R}$ quasars. To answer this question we started a $V L A$ survey of the complete PG quasar sample at $45 \mathrm{GHz}$. Initial results (Baldi et al. in preparation) suggest a positive answer, as also suggested by earlier high frequency studies of AGN (Behar et al. 2015; Doi \& Inoue 2016; Behar et al. 2018; Inoue \& Doi 2018). If such a component indeed originates in the accretion disk corona, then at high enough frequency $(>100 \mathrm{GHz})$ the $\mathrm{mm}$ emission may originate from small enough scales to overlap with the X-ray emitting corona. Correlated $\mathrm{mm}$ and X-ray variability may then be present (Baldi et al. 2015), which will allow to search for the Nuepert effect, a hallmark of magnetic coronal heating in stellar coronae (Neupert 1968; Güdel 2002).

How tightly related are the radio and X-ray emission properties? The radio luminosity versus the X-ray luminosity relation suggests a common coronal origin (LB08). Is there also a corresponding relation between $\alpha_{R}$ and $\alpha_{X}$, as suggested above? An ongoing XMM-Newton survey of the PG quasar sample (Peretz et al. in preparation) will allow us to expand the $\alpha_{R}$ versus $\alpha_{X}$ relation to the $0.3-10 \mathrm{keV}$ range. Such a study will allow addressing various questions. For example, do X-ray absorbed objects also show evidence for radio absorption? Since the two absorption mechanisms are quite different (bound-free versus free-free), such a study can provide new information on the absorber properties (Levinson, Laor, \& Vermeulen 1995; Wilson et al. 1998; Pedlar et al. 1998).

\section{CONCLUSIONS}

An archival study of the $5-8.4 \mathrm{GHz}$ spectral slope, $\alpha_{R}$, of the core radio emission of $25 \mathrm{RQ}$ PGs, reveals a highly significant correlation of $\alpha_{R}$ with $L / L_{\mathrm{Edd}}$. All quasars with $L / L_{\mathrm{Edd}}>$ 0.3 have $\alpha_{R}<-0.5$, and thus their emission is dominated by an extended optically thin emission, on pc scale or larger. Most quasars at $L / L_{\text {Edd }}<0.3$ have $\alpha_{R}>-0.5$ indicative of a compact, sub pc scale synchrotron source.

The RL PGs show a significant correlation of $\alpha_{R}$ with $M_{\mathrm{BH}}$. Most of the PG RLQ with $M_{\mathrm{BH}}>10^{9} M_{\odot}$ have $\alpha_{R}<-0.5$ and their radio is dominated by the extended lobe emission, and most RLQ with $M_{\mathrm{BH}}<10^{9} M_{\odot}$ have $\alpha_{R}>-0.5$, and their radio emission is unresolved.

The different relation of $\alpha_{R}$ with $M_{\mathrm{BH}}$ and $L / L_{\mathrm{Edd}}$ in the RL and RQ PG quasars indicates that RQQ are not just scaled down RLQ, but are rather powered by different mechanisms.

A possible scenario in RQQ is that the extended optically thin emission, which dominates at high $L / L_{\mathrm{Edd}}$, is associated with an outflow which becomes significant at high $L / L_{\mathrm{Edd}}$, such as radiation pressure driven wind. At lower $L / L_{\text {Edd }}$ this extended component does not dominate the emission, and only a compact optically thick component, possibly associated with the coronal emission, is present.

Another interpretation is suggested by the observed $\alpha_{R}$ versus $\alpha_{X}$ relation, which may imply that the driving mech- anism is CME, or a magnetic wind, which become more significant at higher $L / L_{\mathrm{Edd}}$, deplete the corona and produce a more extended magnetised outflow.

This study indicates that the radio emission in RQQ is part of the EV1 set of correlations, and is tightly related to the emission properties at other bands. Given the drastically improving sensitivity of the radio telescopes, from the meter to the sub mm bands, and their vastly higher angular resolution compared to telescopes in other bands, the radio provides a powerful new tool to image a range of physical processes in the innermost parts of RQ AGN.

\section{ACKNOWLEDGEMENTS}

This research was supported by the Israel Science Foundation (grant no. 1561/13). We thank the referee for useful comments on the paper. 

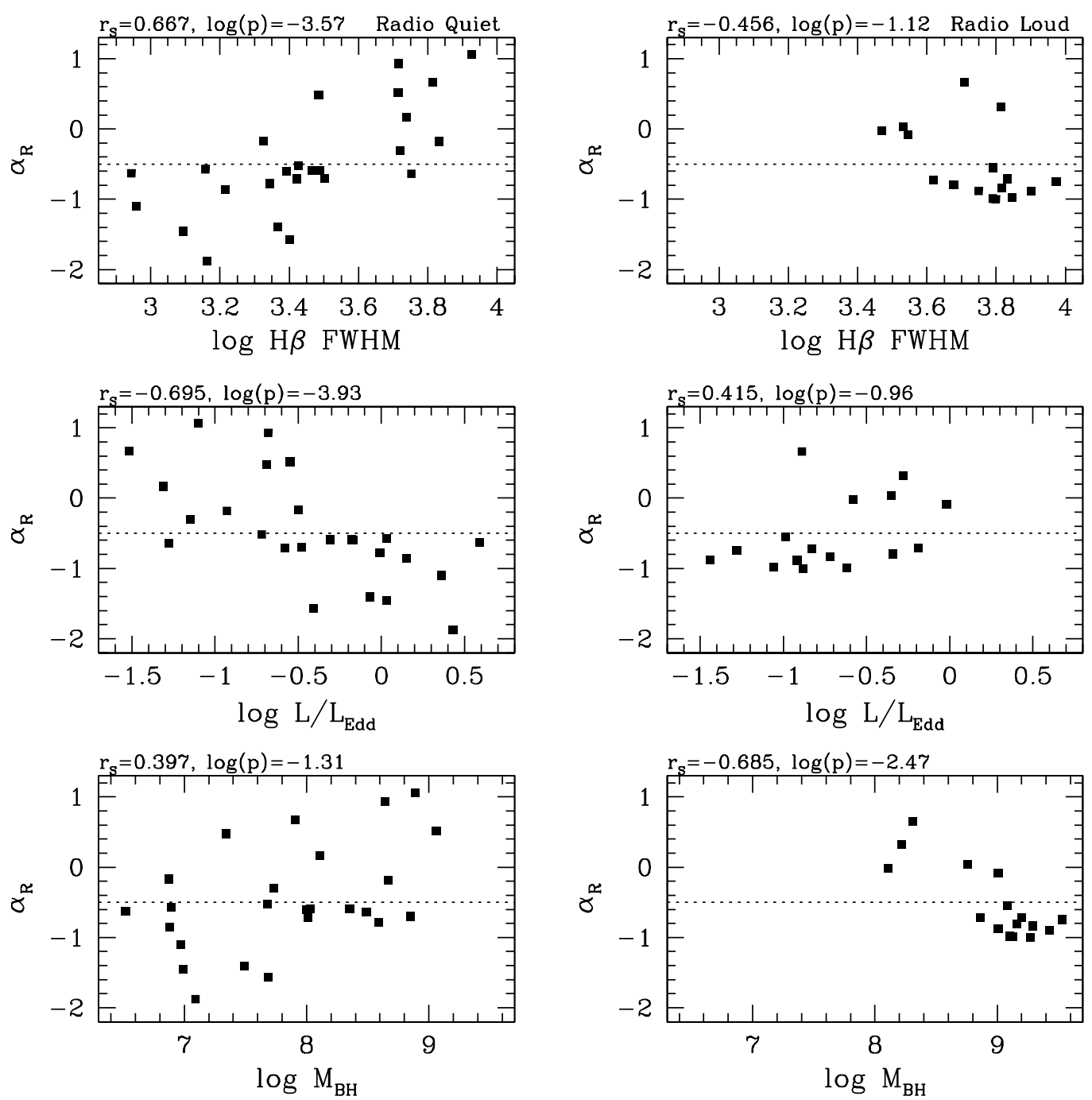

Figure 1. The correlation of $\alpha_{R}$ with the $\mathrm{H} \beta$ FWHM, $L / L_{\mathrm{Edd}}$, and $M_{\mathrm{BH}}$ for the RQ PG quasars (left column), and RL PG quasars (right column). The horizontal dotted line at $\alpha_{R}=-0.5$ separates optically thick emission (above) from optically thin emission (below). The rank order correlation coefficient $\left(r_{\mathrm{S}}\right)$, and the two tailed significance based on the t-distribution $(\mathrm{p})$, are indicated above each panel. Note the highly significant $\mathrm{p}=(1-3) \times 10^{-4}$ correlation of $\alpha_{R}$ with the $\mathrm{H} \beta \mathrm{FWHM}$ and with $L / L_{\mathrm{Edd}}$, and the marginal correlation of $\alpha_{R}$ with $M_{\mathrm{BH}}(\mathrm{p} \simeq 0.05)$ in RQQ. In contrast, in RLQ $\alpha_{R}$ is only marginally correlated, and with an opposite trend, with the $\mathrm{H} \beta$ FWHM and with $L / L_{\mathrm{Edd}}\left(\mathrm{p} \simeq 0.1\right.$ ), and is significantly correlated with $M_{\mathrm{BH}}$ ( $\left.\mathrm{p} \simeq 0.004\right)$, again with an opposite trends. This suggests different radio production mechanisms in RLQ and RQQ, jets versus potentially an $L / L_{\text {Edd }}$ dependent wind (see text).

\section{REFERENCES}

Anderson, J. M., \& Ulvestad, J. S. 2005, ApJ, 627, 674

Antonucci R., Barvainis R., 1988, ApJ, 332, L13

Baldi R. D., Behar E., Laor A., Horesh A., 2015, MNRAS, 454, 4277

Barvainis R., Antonucci R., 1989, ApJS, 70, 257

Barvainis R., Lonsdale C., Antonucci R., 1996, AJ, 111, 1431

Barvainis R., Lonsdale C., 1997, AJ, 113, 144

Barvainis R., Lonsdale C., 1998, AJ, 115, 885

Barvainis R., Lehár J., Birkinshaw M., Falcke H., Blundell K. M., 2005, ApJ, 618, 108

Behar, E., Baldi, R. D., Laor, A., et al. 2015, MNRAS, 451,

\section{7}

Behar E., Vogel S., Baldi R. D., Smith K. L., Mushotzky R. F., 2018, MNRAS, 478, 399

Blundell, K. M., \& Beasley, A. J. 1998, MNRAS, 299, 165

Boroson T. A., 2002, ApJ, 565, 78

Boroson T. A., Green R. F., 1992, ApJS, 80, 109

Boroson T. A., Meyers K. A., 1992, ApJ, 397, 442

Boller T., Brandt W. N., Fink H., 1996, A\&A, 305, 53

Blustin A. J., Fabian A. C., 2009, MNRAS, 396, 1732

Brandt W. N., Mathur S., Elvis M., 1997, MNRAS, 285, L25

Brown M. J. I., et al., 2017, ApJ, 847, 136

Caccianiga, A., Marchã, M. J. M., Thean, A., Dennett-

Thorpe, J. 2001, MNRAS, 328, 867 

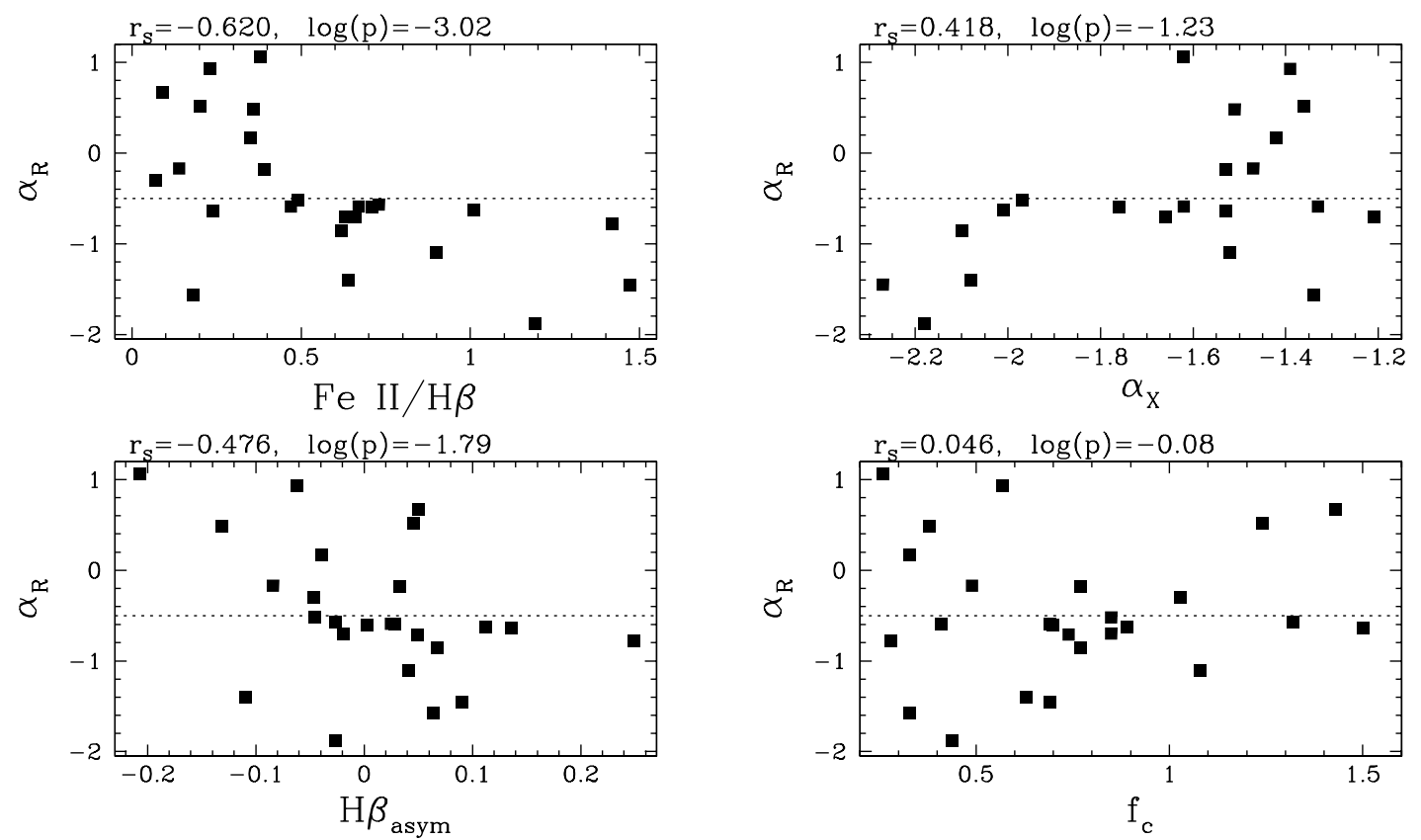

Figure 2. Correlations of $\alpha_{R}$ in the RQ PGs with the FeII/H $\beta$ emission line ratio, with the $\mathrm{H} \beta$ asymmetry parameter (both from Boroson \& Green 1992), the ROSAT $0.2-2 \mathrm{keV}$ slope $\alpha_{X}$, and the $5 \mathrm{GHz}$ emission core dominance parameter $f_{c}$. Flat (optically thick) $\alpha_{R}$ in RQQ is associated with a low FeII/H $\beta$, excess red wing emission in $\mathrm{H} \beta$, and a flat $\alpha_{X}$. These three properties are part of the EV1 set of properties, and are known to correlate with $L / L_{\mathrm{Edd}}$. They may serve as indicators for a radiation pressure driven wind at high $L / L_{\mathrm{Edd}}$, and an associated extended steep radio emission. Note the complete lack of correlation of $\alpha_{R}$ with $f_{\mathrm{c}}$, in contrast with RLQ where core dominated $\left(f_{c}=1\right)$ sources are generally flat, and extended sources are all steep.

Condon J. J., Kellermann K. I., Kimball A. E., Ivezić Ž., Perley R. A., 2013, ApJ, 768, 37

Di Matteo T., Celotti A., Fabian A. C., 1997, MNRAS, 291, 805

Davis S. W., Laor A., 2011, ApJ, 728, 98

Doi, A., Asada, K., Fujisawa, K., et al. 2013, ApJ, 765, 69

Doi A., Inoue Y., 2016, PASJ, 68, 56

Faber S. M., et al., 1997, AJ, 114, 1771

Falcke H., Biermann P. L., 1995, A\&A, 293, 665

Falcke H., Sherwood W., Patnaik A. R., 1996, ApJ, 471, 106

Faucher-Giguère C.-A., Quataert E., 2012, MNRAS, 425, 605

Field G. B., Rogers R. D., 1993, ApJ, 403, 94

Gallimore J. F., Axon D. J., O'Dea C. P., Baum S. A., Pedlar A., 2006, AJ, 132, 546

Green R. F., Schmidt M., Liebert J., 1986, ApJS, 61, 305

Green P. J., Aldcroft T. L., Mathur S., Schartel N., 1997, ApJ, 484, 135

Grupe D., Beuermann K., Mannheim K., Thomas H.-C., 1999, A\&A, 350, 805

Grupe D., 2004, AJ, 127, 1799

Grupe D., Thomas H.-C., Beuermann K., 2001, A\&A, 367,
470

Güdel M., 2002, ARA\&A, 40, 217

Hamann F., et al., 2017, MNRAS, 464, 3431

Heckman T. M., Best P. N., 2014, ARA\&A, 52, 589

Ho L. C., 2008, ARA\&A, 46, 475

Hwang H.-C., Zakamska N. L., Alexandroff R. M., Hamann F., Greene J. E., Perrotta S., Richards G. T., 2018, MNRAS, 477, 830

Inoue Y., Doi A., 2018, arXiv, arXiv:1810.10732

Jester S., et al., 2005, AJ, 130, 873

Jiang Y.-F., Ciotti L., Ostriker J. P., Spitkovsky A., 2010, ApJ, 711, 125

Jones S., McHardy I., Moss D., Seymour N., Breedt E., Uttley P., Körding E., Tudose V., 2011, MNRAS, 412, 2641

Jones S., McHardy I., Maccarone T. J., 2017, MNRAS, 465, 1336

Kellermann K. I., Sramek R., Schmidt M., Shaffer D. B., Green R., 1989, AJ, 98, 1195

Kellermann K. I., Sramek R. A., Schmidt M., Green R. F., Shaffer D. B., 1994, AJ, 108, 1163

Kellermann K. I., Condon J. J., Kimball A. E., Perley R. A., Ivezić Ž., 2016, ApJ, 831, 168 


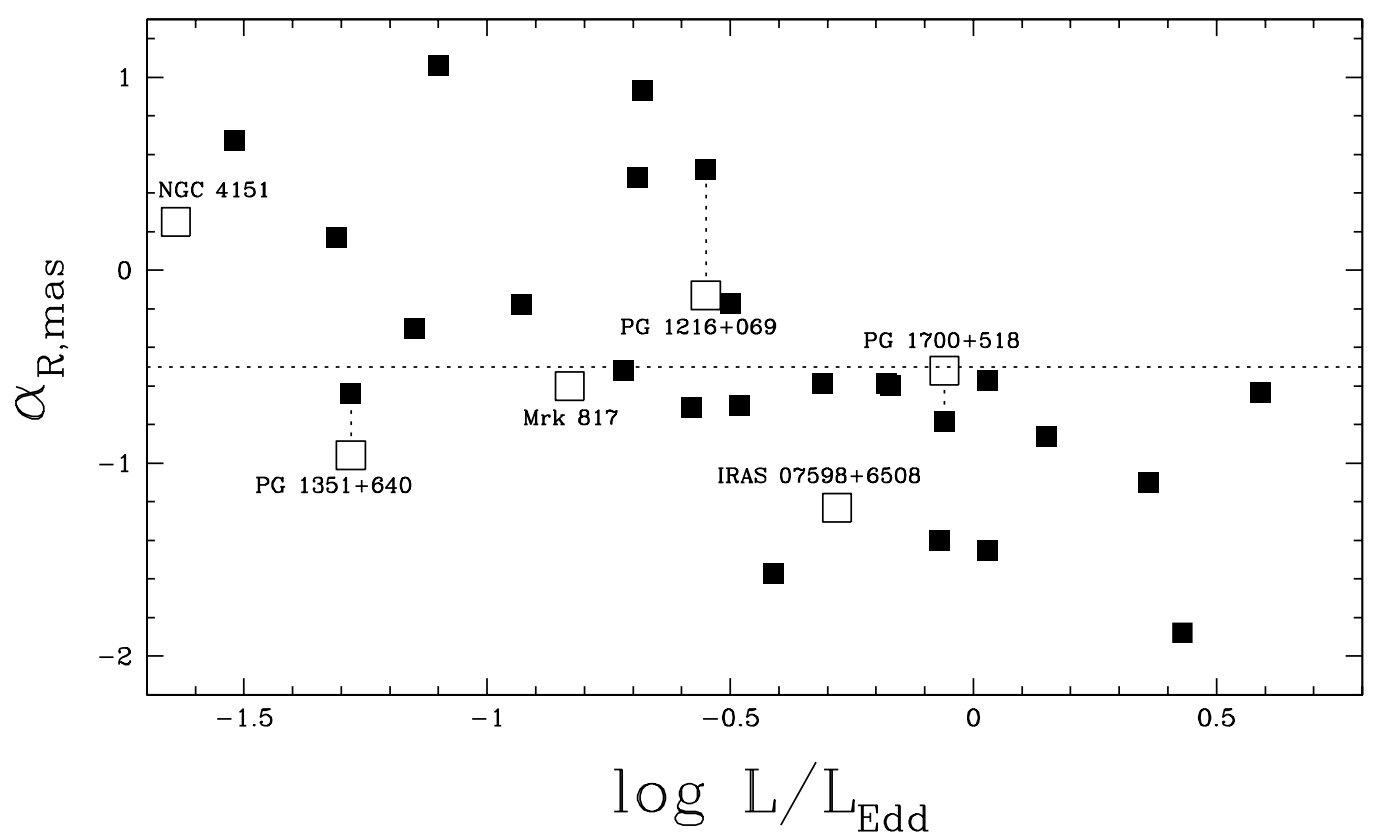

Figure 3. VLBI observations of the mas scale radio slope $\alpha_{\text {mas }}$ (open squares) versus $L / L_{\mathrm{Edd}}$, available for three of the $25 \mathrm{RQ} P G s$, and for three additional type 1 AGN with $\log L / L_{\text {Edd }}>-2$, on top of the distribution of $\alpha_{R}$ (filled squares) versus $L / L_{\mathrm{Edd}}$ (Fig. 1 ). The mas, pc scale, emission appears to follow (with some scatter) the $\alpha_{R}$ versus $L / L_{\mathrm{Edd}}$ relation, observed at a resolution of a fraction of kpc.

Kennicutt R. C., Evans N. J., 2012, ARA\&A, 50, 531 Kukula M. J., Pedlar A., Baum S. A., O’Dea C. P., 1995, MNRAS, 276, 1262

Kukula M. J., Dunlop J. S., Hughes D. H., Rawlings S., 1998, MNRAS, 297, 366

Laor A., Fiore F., Elvis M., Wilkes B. J., McDowell J. C., 1994, ApJ, 435, 611

Laor A., Fiore F., Elvis M., Wilkes B. J., McDowell J. C., 1997, ApJ, 477, 93

Laor A., Brandt W. N., 2002, ApJ, 569, 641

Laor A., Behar E., 2008, MNRAS, 390 , 847 (LB08)

Leipski C., Falcke H., Bennert N., Hüttemeister S., 2006, A\&A, 455, 161

Levinson A., Laor A., Vermeulen R. C., 1995, ApJ, 448, 589

Merloni A., Di Matteo T., Fabian A. C., 2000, MNRAS, 318, L15
Middelberg E., et al., 2004, A\&A, 417, 925

Miller P., Rawlings S., Saunders R., 1993, MNRAS, 263, 425

Moran E. C., 2000, arXiv:astro-ph/0005149

Mundell C. G., Wilson A. S., Ulvestad J. S., Roy A. L., 2000, ApJ, 529, 816

Mundell, C. G., Ferruit, P., Nagar, N., \& Wilson, A. S. 2009, ApJ, 703, 802

Neupert W. M., 1968, ApJ, 153, L59

Nims J., Quataert E., Faucher-Giguère C.-A., 2015, MNRAS, 447, 3612

O'Dea C. P., 1998, PASP, 110, 493

Orienti M., Prieto M. A., 2010, MNRAS, 401, 2599

Padovani P., Miller N., Kellermann K. I., Mainieri V., Rosati P., Tozzi P., 2011, ApJ, 740, 20

Panessa, F., \& Giroletti, M. 2013, MNRAS, 432, 1138

Pedlar A., Fernandez B., Hamilton N. G., Redman M. P., 
Table 1. Radio Quiet PG Quasars

\begin{tabular}{|c|c|c|c|c|c|c|c|c|c|c|c|c|}
\hline $\begin{array}{r}\text { Name } \\
(1)\end{array}$ & $\begin{array}{l}L_{\mathrm{bol}} \\
(2)\end{array}$ & $\begin{array}{l}\mathrm{L}_{\mathrm{R}} \\
(3)\end{array}$ & $\begin{array}{l}R_{X} \\
(4)\end{array}$ & $\begin{array}{l}\text { W } \\
(5)\end{array}$ & $\begin{array}{l}M_{\mathrm{BH}} \\
(6)\end{array}$ & $\begin{array}{r}\frac{L}{L_{\mathrm{Edd}}} \\
(7)\end{array}$ & $\begin{array}{l}f_{5} \\
(8)\end{array}$ & $\begin{array}{l}f_{8.5} \\
(9)\end{array}$ & $\begin{array}{l}\text { Ref. } \\
(10)\end{array}$ & $\begin{array}{r}\alpha_{R} \\
(11)\end{array}$ & $\begin{array}{r}\alpha_{X} \\
(12)\end{array}$ & $\begin{array}{l}\text { Ref. } \\
(13)\end{array}$ \\
\hline $003+199$ & 45.13 & 38.36 & -5.44 & 1640 & 6.88 & 0.15 & 3.03 & $1.89 / \mathrm{A}$ & Ku95 & -0.86 & -2.10 & G01 \\
\hline $0049+171$ & $44.68^{c}$ & 38.27 & -5.78 & 5250 & 7.73 & -1.15 & 0.66 & $0.56 / \mathrm{A} / \mathrm{B}$ & $\mathrm{Ba} 05$ & -0.30 & $\mathrm{x}$ & \\
\hline $0050+124$ & 45.12 & 38.97 & -5.21 & 1240 & 6.99 & 0.03 & 1.80 & $0.81 / \mathrm{A}$ & Ku95 & -1.45 & -2.27 & Sc96 \\
\hline $0052+251$ & 46.06 & 39.35 & -5.58 & 5200 & 8.64 & -0.68 & 0.42 & $0.70 / \mathrm{A}$ & Ku98 & 0.93 & -1.39 & W96 \\
\hline $0157+001$ & 45.93 & 40.13 & -4.10 & 2460 & 8.00 & -0.17 & 5.58 & $4.00 / \mathrm{A}$ & Ku98 & -0.60 & -1.76 & UM96 \\
\hline $0804+761$ & 45.82 & 39.88 & -4.90 & 3070 & 8.03 & -0.31 & 0.97 & $0.70 / \mathrm{A}$ & Ku98 & -0.59 & -1.33 & WF93 \\
\hline $0921+525$ & 44.47 & 38.62 & -4.90 & 2120 & 6.87 & -0.50 & 1.87 & $1.70 / \mathrm{A}$ & Ku98 & -0.17 & -1.47 & G01 \\
\hline $1011-040$ & 45.02 & 38.09 & -4.51 & 1440 & 6.89 & 0.03 & 0.37 & $0.27 / \mathrm{B}$ & $\mathrm{Ar}$ & -0.57 & $\mathrm{x}$ & \\
\hline $1012+008$ & 45.53 & 39.50 & -4.56 & 2640 & 8.01 & -0.58 & 0.74 & $0.50 / \mathrm{A}$ & Ku98 & -0.71 & -1.66 & W96 \\
\hline $1116+215$ & 46.27 & 40.20 & -4.63 & 2920 & 8.35 & -0.18 & 1.94 & $1.40 / \mathrm{A}$ & Ku98 & -0.59 & -1.62 & G01 \\
\hline $1149-110$ & 44.75 & 38.80 & -5.03 & 3060 & 7.34 & -0.69 & 1.00 & $1.30 / \mathrm{A}$ & Ku98 & 0.48 & -1.51 & W96 \\
\hline $1216+069$ & 46.61 & 40.62 & -4.48 & 5190 & 9.06 & -0.55 & $9.08^{a}$ & $12.1 / \mathrm{C}$ & Ba96 & 0.52 & -1.36 & L97 \\
\hline $1351+640$ & 45.31 & 40.29 & -3.24 & 5660 & 8.49 & -1.28 & $7.02^{a}$ & $3.45 / \mathrm{C}^{b}$ & Ba96 & -0.64 & -1.53 & W96 \\
\hline $1404+226$ & 45.21 & 38.67 & -5.10 & 880 & 6.52 & 0.59 & 0.89 & $0.63 / \mathrm{C}$ & Ba96 & -0.63 & -2.01 & UM96 \\
\hline $1411+442$ & 45.06 & 38.50 & -4.22 & 2670 & 7.68 & -0.72 & 0.52 & $0.39 / \mathrm{C} / \mathrm{D}$ & BL97 & -0.52 & -1.97 & L97 \\
\hline $1426+015$ & 45.84 & 39.30 & -5.28 & 6820 & 8.67 & -0.93 & $0.84^{a}$ & $0.76 / \mathrm{C}$ & Ba96 & -0.18 & -1.53 & WF93 \\
\hline $1440+356$ & 45.62 & 38.94 & -5.60 & 1450 & 7.09 & 0.43 & 0.73 & $0.26 / \mathrm{A}$ & $\mathrm{Ar}$ & -1.88 & -2.18 & G01 \\
\hline $1448+273$ & $45.43^{c}$ & 38.58 & -5.28 & 910 & $6.97^{c}$ & 0.36 & $1.34^{a}$ & $0.73 / \mathrm{C}$ & Ba96 & -1.10 & -1.52 & G01 \\
\hline $1501+106$ & 44.90 & 38.92 & -4.72 & 5470 & 6.97 & -1.31 & 0.50 & $0.55 / \mathrm{A}$ & Ku95 & 0.17 & -1.42 & G01 \\
\hline $1612+261$ & 45.38 & 40.02 & -4.51 & 2520 & 7.69 & -0.41 & 1.66 & $0.70 / \mathrm{A}$ & Ku98 & -1.57 & -1.34 & Sc96 \\
\hline $1613+658$ & 45.89 & 39.55 & -5.34 & 8450 & 8.89 & -1.10 & 0.78 & $1.40 / \mathrm{A}$ & Ku98 & 1.06 & -1.62 & G01 \\
\hline $1700+518$ & $46.63^{a}$ & 40.75 & $-1.74^{c}$ & 2210 & $8.59^{c}$ & -0.06 & $1.32^{e}$ & $0.86 / \mathrm{A}^{e}$ & Ru18 & -0.78 & $\mathrm{x}$ & \\
\hline $2112+059$ & 46.47 & 40.51 & -3.36 & 3190 & 8.85 & -0.48 & 0.76 & $0.52 / \mathrm{B} / \mathrm{D}$ & $\mathrm{Ba} 05$ & -0.70 & -1.21 & W96 \\
\hline $2130+099$ & 45.52 & 39.00 & -5.35 & 2330 & 7.49 & -0.07 & 1.30 & $0.60 / \mathrm{A}$ & Ku98 & -1.40 & -2.08 & G01 \\
\hline $2304+042$ & 44.49 & 38.14 & -5.75 & 6500 & 7.91 & -1.52 & $\mathrm{~d}$ & $0.65 / \mathrm{A} / \mathrm{D}$ & $\mathrm{Ba} 05$ & 0.67 & $\mathrm{x}$ & \\
\hline
\end{tabular}

Note. - Col. (1): PG object designation; col. (2): Log of bolometric luminosity in erg $\mathrm{s}^{-1}$ (Davis \& Laor 2011); col. (3) Log of the radio luminosity in observed frame $5 \mathrm{GHz}$ in erg s$^{-1}$ (Kellermann et al. 1989); col. 4) Log of the radio to X-ray luminosities, taken from LB08 (Table 1 there); col. (5) The broad H $\beta$ line FWHM in km s${ }^{-1}$ (Boroson \& Green 1992); col. (6) Log of the BH mass in units of $M_{\odot}$ (Davis \& Laor 2011); col. (7) Log of the luminosity in Eddington units, based on columns (2) and (6); col. (8) The unresolved (i.e. peak) flux at $4.89 \mathrm{GHz}$, observed in the A configuration ; col. (9) The unresolved (i.e. peak) flux at $8.4 \mathrm{GHz}$, and the configuration used; col. (10) The reference for the $8.4 \mathrm{GHz}$ observation; col. (11) The value of $\alpha_{R}$ based on columns (9) and (10); col. (12) The ROSAT $0.2-2 \mathrm{keV}$ slope, $\alpha_{X}$, corrected for the foreground Galactic absorption; col. (13) The reference for the value of $\alpha_{X}$.

a - The flux at the D configuration in Barvainis, Lonsdale, \& Antonucci (1996) and in Kellermann et al. (1989) are significantly different, which indicates variability. We therefore measure $\alpha_{R}$ using the lower resolution but nearly simultaneous observations of both bands in Barvainis, Lonsdale, \& Antonucci (1996).

b - Flux at $14.9 \mathrm{GHz}$.

c - Estimated as $9 \nu L_{\nu}(5100 \AA)$, based on the mean SED of Richards et al. (2006), where $\nu L_{\nu}(5100 \AA)$ is from Vestergaard \& Peterson (2006).

c - Taken from Vestergaard \& Peterson (2006).

d - The Barvainis et al. (2005) observations indicate large variability (more than a factor of two in less than half a year). We therefore use the slope provided by Barvainis et al. (2005), derived from lower resolution, but nearly simultaneous observations.

e - Peak core fluxes (western source) reported in Runnoe, Gültekin, \& Rupke (2018).

$\mathrm{x}$ - No published ROSAT detection, or the $\mathrm{S} / \mathrm{N}$ is too low.

References: Ar - An archive search; Ba96 - Barvainis, Lonsdale, \& Antonucci (1996); Ba05 - Barvainis et al. (2005); BL97 - Barvainis \& Lonsdale (1997); G01 - Grupe, Thomas, \& Beuermann (2001); Ku95 - Kukula et al. (1995); Ku98 - Kukula et al. (1998); L97 - Laor et al. (1997); Ru18 - Runnoe, Gültekin, \& Rupke (2018); Sc96 - Schartel et al. (1996); UM96 - Ulrich-Demoulin \& Molendi (1996); W96 - Wang, Brinkmann, \& Bergeron (1996); WF93 - Walter \& Fink (1993).

Dewdney P. E., 1998, MNRAS, 300, 1071

Piconcelli E., Jimenez-Bailón E., Guainazzi M., Schartel N., Rodríguez-Pascual P. M., Santos-Lleó M., 2005, A\&A, 432,15

Porquet D., Reeves J. N., O'Brien P., Brinkmann W., 2004, A\&A, 422, 85

Raginski I., Laor A., 2016, MNRAS, 459, 2082 (RL16)

Reeves J. N., Turner M. J. L., 2000, MNRAS, 316, 234
Richards G. T., et al., 2006, ApJS, 166, 470

Runnoe J. C., Gültekin K., Rupke D. S. N., 2018, ApJ, 852,8

Rybicki G. B., Lightman A. P., 1979, Radiative Processes in Astrophysics

Sani E., Lutz D., Risaliti G., Netzer H., Gallo L. C., Trakhtenbrot B., Sturm E., Boller T., 2010, MNRAS, 403, 1246 Schartel N., Walter R., Fink H. H., Truemper J., 1996, 
Table 2. Radio Loud PG Quasars

\begin{tabular}{cllllllr}
\hline $\begin{array}{c}\text { Name } \\
(1)\end{array}$ & $\begin{array}{l}L_{\text {bol }} \\
(2)\end{array}$ & $\begin{array}{l}\mathrm{L}_{\mathrm{R}} \\
(3)\end{array}$ & $\begin{array}{l}R_{X} \\
(4)\end{array}$ & $\begin{array}{l}\mathrm{W} \\
(5)\end{array}$ & $\begin{array}{l}M_{\mathrm{BH}} \\
(6)\end{array}$ & $\begin{array}{l}L / L_{\mathrm{Edd}} \\
(7)\end{array}$ & $\begin{array}{r}\alpha_{R} \\
(8)\end{array}$ \\
\hline $0003+158$ & 46.92 & 42.99 & -2.56 & 4760 & 9.16 & -0.34 & -0.80 \\
$0007+106$ & 45.52 & 41.93 & -2.56 & 5100 & 8.31 & -0.89 & 0.66 \\
$1004+130$ & $46.49^{a}$ & 42.70 & -1.78 & 6300 & $9.27^{b}$ & -0.88 & -1.00 \\
$1048-090$ & 46.57 & 42.97 & -2.19 & 5620 & 9.01 & -1.44 & -0.88 \\
$1100+772$ & 46.61 & 42.92 & -2.32 & 6160 & 9.13 & -0.62 & -0.99 \\
$1103-006$ & 46.19 & 42.88 & -2.02 & 6190 & 9.08 & -0.99 & -0.55 \\
$1226+023$ & 47.09 & 43.97 & -1.70 & 3520 & 9.01 & -0.02 & -0.09 \\
$1302-102$ & 47.04 & 42.99 & -2.10 & 3400 & 8.76 & -0.35 & 0.04 \\
$1309+355$ & 45.63 & 41.05 & -2.84 & 2940 & 8.11 & -0.58 & -0.02 \\
$1425+267$ & 46.35 & 41.82 & -2.39 & 9410 & 9.53 & -1.28 & -0.75 \\
$1512+370$ & 47.11 & 42.63 & -2.40 & 6810 & 9.20 & -0.19 & -0.71 \\
$1545+210$ & 46.14 & 42.91 & -2.23 & 7030 & 9.10 & -1.06 & -0.98 \\
$1704+608$ & 46.67 & 43.29 & -1.35 & 6560 & 9.29 & -0.72 & -0.84 \\
$2209+184$ & 46.02 & 41.45 & -2.49 & 6500 & 8.22 & -0.28 & 0.32 \\
$2251+113$ & 46.13 & 43.06 & -1.03 & 4160 & 8.86 & -0.83 & -0.72 \\
$2308+098$ & 46.61 & 42.76 & -2.59 & 7970 & 9.43 & -0.92 & -0.89 \\
\hline
\end{tabular}

Note. - Cols. (1)-(7): As in Table 1; col. (8) From Falcke, Sherwood, \& Patnaik (1996).

a - Estimated as $9 \nu L_{\nu}(5100 \AA)$, based on the mean SED of Richards et al. (2006), where $\nu L_{\nu}(5100 \AA)$ is from Vestergaard \& Peterson (2006).

b - From Vestergaard \& Peterson (2006).

A\&A, 307, 33

Schmidt M., Green R. F., 1983, ApJ, 269, 352

Shemmer O., Netzer H., 2002, ApJ, 567, L19

Shemmer O., Netzer H., Maiolino R., Oliva E., Croom S.,

Corbett E., di Fabrizio L., 2004, ApJ, 614, 547

Shemmer O., Brandt W. N., Netzer H., Maiolino R., Kaspi

S., 2006, ApJ, 646, L29

Shin J., nagao T., Woo J.-H., 2017, ApJ, 835, 24

Steenbrugge K. C., Jolley E. J. D., Kuncic Z., Blundell

K. M., 2011, MNRAS, 413, 1735

Sulentic J. W., Marziani P., Dultzin-Hacyan D., 2000,

ARA\&A, 38, 521

Tabatabaei F. S., et al., 2017, ApJ, 836, 185

Terashima Y., Wilson A. S., 2003, ApJ, 583, 145

Ulvestad J. S., Antonucci R. R. J., Barvainis R., 2005, ApJ, 621,123

Ulvestad J. S., Wong D. S., Taylor G. B., Gallimore J. F., Mundell C. G., 2005, AJ, 130, 936

Ulrich-Demoulin M.-H., Molendi S., 1996, ApJ, 457, 77

Vestergaard M., Peterson B. M., 2006, ApJ, 641, 689

Walter R., Fink H. H., 1993, A\&A, 274, 105

Wang T., Brinkmann W., Bergeron J., 1996, A\&A, 309, 81

White S. V., Jarvis M. J., Häußler B., Maddox N., 2015,

MNRAS, 448, 2665

Wills B. J., Browne I. W. A., 1986, ApJ, 302, 56

Wills B. J., Laor A., Brotherton M. S., Wills D., Wilkes B. J., Ferland G. J., Shang Z., 1999, ApJ, 515, L53

Wilson A. S., Colbert E. J. M., 1995, ApJ, 438, 62

Wilson A. S., et al., 1998, ApJ, 505, 587

Wrobel, J. M. 2000, ApJ, 531, 716

Yang J., Wu F., Paragi Z., An T., 2012, MNRAS, 419, L74

Zakamska N. L., Greene J. E., 2014, MNRAS, 442, 784

Zakamska N. L., et al., 2016, MNRAS, 455, 4191 